\title{
Mapping Body Shape Genes through Shape Mapping
}

\section{Ningtao Wang, Yaqun Wang, Zhong Wang, Han Hao and Rongling $\mathbf{W u}^{*}$}

Center for Statistical Genetics, The Pennsylvania State University, Hershey, PA 17033, USA

\section{Body Shape as a Predictor of Human Health}

It has been well known that human body shape, as a complex phenomenon with sophisticated detail and function, is associated with human health [1-3]. Body shape has been commonly described by a parameter, called Body Mass Index (BMI), which is defined as an individual's body mass divided by the square of his or her height. BMI has long been criticized, because it can only measure the overall adiposity of a body, failing to take into account body composition. For example, two people who are equal in height and body mass (and therefore in BMI) may differ dramatically in body shape (Figure 1), as they have different places to store fat, producing different health outlooks. Therefore, one expects the quantitative power of BMI for distinguishing body shapes to be much lower than it should be.

Waist-Hip Ratio (WHR) is of particular interest as a measure of body fat distribution, and is thought to be a predictor of metabolic consequences, independent of BMI. WHR can be roughly used to classify people into apple-shaped type, carrying their excess weight around their waists, and pear-shaped type, with fat lower down around their hips, thighs and buttocks (Figure 2). Increasing WHR is associated with adverse metabolic risk causing diabetes, hypertension and dyslipidemia [4]. In other words, pear shapes tend to have a lower risk for heart disease and type 2 diabetes, when compared to the appleshaped population. In a recent genome-wide association study by Heid et al. [5], 13 new genes have been found to affect WHR, seven of which exhibited pronounced sexual dimorphism, all with a stronger effect in women than men. All these detected genes are involved in chromosomal regions controlling cholesterol, insulin, and insulin resistance, linked to the development of diabetes.

Although this study, along with others [6,7], has refreshed our view of the genetic control of body shape, there is still a long journey towards a detailed understanding of the biological mechanisms involved in the pattern of where our body stores fat. A major reason is that WHR contains little information about the spatial distribution of shape changes across the body, and thereby, why fat accumulates in different places is far less clear based on WHR alone. Now, a new computational model has been available to map specific genes associated with body shape in a precise manner [8]. This model, called shape mapping, is the

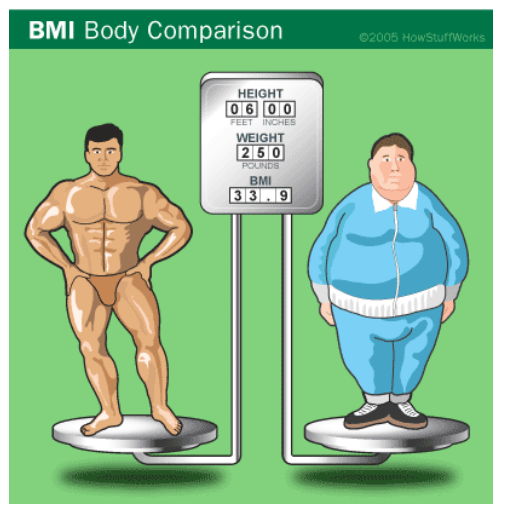

Figure 1: Two people of the same BMI display different body shapes. Downloaded from http://srxawordonhealth.com/tag/belly-fat/. integration of two seemingly disjoined areas, geometric morphometrics and genetic mapping.

\section{Shape Mapping}

An early attempt to quantitatively describe the mechanism of shape change was made by D’Arcy Thompson [9]. Inspired by Thompson's work, a revolutionary approach, called Geometric Morphometrics (GM), has been put forward in the early 1980's [10,11], to study shape variation by determining, analyzing and connecting meaningful anatomical landmarks of the organism. This approach detects shape changes in a Cartesian coordinate system and extracts the geometric information about the relative positions of landmarks. A set of landmarks that represent a shape of an object, distributed homogeneously on the organism, and bearing some biological meaning are chosen. Shape is aligned, described and compared through filtering location, scale and rotational effects, out from an object [12]. Several methods including a procrustes analysis [13], have been proposed for shape alignment.

If biologically meaningful landmarks cannot be identified, an alternative, called outline analysis, has been developed and can be used [14]. By recording a high number of positions along the contour of the object studied, outline analysis uses a mathematical function to fit these ordered points. The differences between shapes can be characterized through the analysis of the mathematical descriptors fitted to them. There are many approaches for outline analysis, including "Eigenshape" analysis, standard Fourier descriptors and Elliptic Fourier analysis.

For the landmark-based approach, a multivariate statistical analysis, such as Principal Component Analysis (PCA), is used to decompose the original high-dimensional space to a space of reduced dimension. Such an analysis removes redundant information by mapping the highdimensional data to the subspace that best account for the distribution

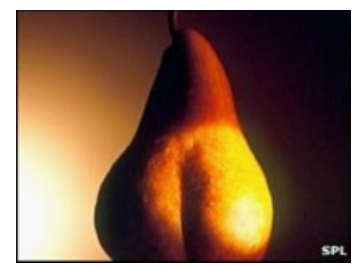

Figure 2: A typical body shape like a pear. Downloaded from http://www. bbc.co.uk/news/health-11503875.

${ }^{*}$ Corresponding author: Rongling Wu, Center for Statistical Genetics, The Pennsylvania State University, Hershey, PA 17033, USA, E-mail: rwu@phs.psu.edu

Received November 23, 2012; Accepted November 26, 2012; Published December 01, 2012

Citation: Wang N, Wang Y, Wang Z, Hao H, Wu R (2012) Mapping Body Shape Genes through Shape Mapping. J Biom Biostat 3:e121. doi:10.4172/2155$6180.1000 \mathrm{e} 121$

Copyright: (C) 2012 Wang N, et al. This is an open-access article distributed under the terms of the Creative Commons Attribution License, which permits unrestricted use, distribution, and reproduction in any medium, provided the original author and source are credited. 
of the original pattern. Each axis on a PCA plot presents an eigenvector of the covariance matrix among shape variables. The first axis contributes to maximum variation in the sample, whereas further axes contribute in other ways, in which the samples vary. Shape mapping intends to map Quantitative Trait Loci (QTLs) for shape variation, by incorporating axis values as phenotypic traits into a mixture model, in which each component corresponds to a different QTL genotype. Difference in these axis values among QTL genotypes are tested and estimated, which can assess the effects of QTLs on detailed structures of a shape. Statistical techniques allow these QTL effects to be visualized as configurations of landmarks, back in the original space of body shape.

Different from the landmark-based approach, outline analysis incorporates mathematical aspects of shape (usually some form of Fourier analysis) into the mixture model, in a way similar to functional mapping [15]. By estimating and testing mathematical parameters that define shape, the dynamic effect of QTLs on shape variation can be quantified. In a recent study, Wang et al. [16] proposes a state-of-theart procedure for examining the control patterns of specific QTLs on the origin, properties and functions of biological shape, using outline analysis. Fourier coefficients that describe QTL effects on shape variation can be transformed back to the physical space of body shape, and visualized as outlines. Their statistical framework can address many integrative biological and genetic questions and challenges in shape variation, faced by the life sciences community.

\section{Outlook to Detect Genes for Apple and Pear Shapes}

Although it has been aware that different body shapes exist, only now have we become interested in their causes and associations with health risks. The latest discovery is that the pattern of where we are genetically and hormonally predisposed to store fat can produce remarkable effects on health risks. An increasing body of study uses powerful Genome-Wide Association Studies (GWAS) to characterize the genetic architecture of body shape, described by body mass index and waist-hip ratio. These two parameters, however, have proven to be too simple to capture the sophisticated detail and function of human body. A newly developed computational model, shape mapping [14,15], has shown its potential to reveal the developmental mechanisms that form different shapes, apple or pear, and therefore, should find its immediate implications for precisely identifying apple- or pear-shape genes. There is no doubt that its integration with GWAS will change our view of the genetic control of body shape, which in turn may help design new therapies or drugs for obesity prevention.

\section{Acknowledgements}

This work is supported by NSF/IOS-0923975, Changjiang Scholars Award, and "Thousand-person Plan" Award.

\section{References}

1. Wang Y, Rimm EB, Stampfer MJ, Willett WC, Hu FB (2005) Comparison of abdominal adiposity and overall obesity in predicting risk of type 2 diabetes among men. Am J Clin Nutr 81: 555-563.

2. Canoy D (2008) Distribution of body fat and risk of coronary heart disease in men and women. Curr Opin Cardiol 23: 591-598.

3. Pischon T, Boeing H, Hoffmann K, Bergmann M, Schulze MB, et al. (2008) General and abdominal adiposity and risk of death in Europe. N Engl $\mathrm{J}$ Med 359: 2105-2120.

4. Snijder MB, Dekker JM, Visser M, Bouter LM, Stehouwer CD, et al. (2003) Associations of hip and thigh circumferences independent of waist circumference with the incidence of type 2 diabetes: the Hoorn Study. Am J Clin Nutr 77: 1192-1197.

5. Heid IM, Jackson AU, Randall JC, Winkler TW, Qi L, et al. (2010) Meta-analysis identifies 13 new loci associated with waist-hip ratio and reveals sexual dimorphism in the genetic basis of fat distribution. Nat Genet 42: 949-960.

6. Lindgren CM, Heid IM, Randall JC, Lamina C, Steinthorsdottir V, et al. (2009) Genome-wide association scan meta-analysis identifies three loci influencing adiposity and fat distribution. PLoS Genet 5: e1000508.

7. Emilsson V, Thorleifsson G, Zhang B, Leonardson AS, Zink F, et al. (2008) Genetics of gene expression and its effect on disease. Nature 452: 423 428.

8. Fu GF, Bo WB, Pang XM, Wang Z, Chen L, et al. (2012) Mapping shape QTLs using a radius-entroid-contour model. Heredity (in press).

9. Thompson D'Arcy W (1917) On Growth and Form. Cambridge University Press, New York.

10. Bookstein FL (1991) Morphometric Tools for Landmark Data: Geometry and Biology. Cambridge University Press, New York, USA.

11. Adams DC, Rohlf FJ, Slice DE (2004) Geometric morphometrics: ten years of progress following the 'revolution'. Ital J Zool 71: 5-16.

12. Kendall DG (1984) Shape manifolds, procrustean metrics, and complex projective spaces. Bull London Math Soc 16: 81-121.

13. Zelditch ML, Swiderski DL, Sheets HD, Fink WL (2004) Geometric Morphometrics for Biologists. (1stedn), Elsevier Academic Press, Amsterdam.

14. Rohlf FJ (1990) Fitting curves to outlines. In: Proceedings of the Michigan Morphometrics Workshop, edited by Rohlf FJ, Bookstein FJ, The University of Michigan Museum of Zoology, Ann Arbor, Michigan.

15. Wu R, Lin M (2006) Functional mapping - how to map and study the genetic architecture of dynamic complex traits. Nat Rev Genet 7: 229-237.

16. Wang Z, Bo WH, Xu F, Fu GF, Sui YH, et al. (2012) Shape mapping: Genetic mapping meets geometric morphometrics. Brief Bioinform (in review). 